Lecture Notes in Computational Vision and Biomechanics 12

Rui Lima

Yohsuke Imai

Takuji Ishikawa

Mónica S. N. Oliveira Editors

Visualization and

Simulation of

Complex Flows

in Biomedical

Engineering

Springer 


\section{Editors}

Rui Lima

ESTiG, Polytechnic Institute of Bragança

Bragança

Portugal

and

CEFT, Faculdade de Engenharia da

Universidade do Porto (FEUP)

Porto

Portugal

Yohsuke Imai

Department of Biomedical Engineering and Robotics, Graduate School

of Biomedical Engineering

Tohoku University

Sendai

Japan
Takuji Ishikawa

Department of Bioengineering and Robotics, Graduate School of Engineering

Tohoku University

Sendai

Japan

Mónica S. N. Oliveira

James Weir Fluids Lab, Department of

Mechanical and Aerospace Engineering

University of Strathclyde

Glasgow

UK
ISSN 2212-9391

ISBN 978-94-007-7768-2

DOI 10.1007/978-94-007-7769-9

Springer Dordrecht Heidelberg New York London

Library of Congress Control Number: 2013949598
ISSN 2212-9413 (electronic)

ISBN 978-94-007-7769-9 (eBook)

(C) Springer Science+Business Media Dordrecht 2014

This work is subject to copyright. All rights are reserved by the Publisher, whether the whole or part of the material is concerned, specifically the rights of translation, reprinting, reuse of illustrations, recitation, broadcasting, reproduction on microfilms or in any other physical way, and transmission or information storage and retrieval, electronic adaptation, computer software, or by similar or dissimilar methodology now known or hereafter developed. Exempted from this legal reservation are brief excerpts in connection with reviews or scholarly analysis or material supplied specifically for the purpose of being entered and executed on a computer system, for exclusive use by the purchaser of the work. Duplication of this publication or parts thereof is permitted only under the provisions of the Copyright Law of the Publisher's location, in its current version, and permission for use must always be obtained from Springer. Permissions for use may be obtained through RightsLink at the Copyright Clearance Center. Violations are liable to prosecution under the respective Copyright Law. The use of general descriptive names, registered names, trademarks, service marks, etc. in this publication does not imply, even in the absence of a specific statement, that such names are exempt from the relevant protective laws and regulations and therefore free for general use.

While the advice and information in this book are believed to be true and accurate at the date of publication, neither the authors nor the editors nor the publisher can accept any legal responsibility for any errors or omissions that may be made. The publisher makes no warranty, express or implied, with respect to the material contained herein.

Printed on acid-free paper

Springer is part of Springer Science+Business Media (www.springer.com) 


\title{
Flow of Red Blood Cells Suspensions Through Hyperbolic Microcontractions
}

\author{
Vera Faustino, Diana Pinho, Tomoko Yaginuma, \\ Ricardo C. Calhelha, Geyong M. Kim, Sergio Arana, \\ Isabel C. F. R. Ferreira, Mónica S. N. Oliveira and Rui Lima
}

\begin{abstract}
The present study uses a hyperbolic microchannel with a low aspect ratio (AR) to investigate how the red blood cells (RBCs) deform under conditions of both extensional and shear induced flows. The deformability is presented by the
\end{abstract}

V. Faustino $(\Phi) \cdot$ D. Pinho - T. Yaginuma $\cdot$ R. Lima

ESTiG, Polytechnic Institute of Bragança, C. Sta. Apolónia 5301-857 Bragança, Portugal e-mail: verafaustino@ipb.pt

D. Pinho

e-mail: diana@ipb.pt

T. Yaginuma

e-mail: tomoko@ipb.pt

R. Lima

e-mail: ruimec@ipb.pt

G. M. Kim * S. Arana

CEIT and Tecnun, University of Navarra, Paseo de Manuel Lardizábal N 1520.018

Donostia, San Sebastián, Spain

e-mail: gmkim@ceit.es

S. Arana

e-mail: sarana@ceit.es

M. S. N. Oliveira

James Weir Fluids Lab, Department of Mechanical and Aerospace Engineering,

University of Strathclyde, Glasgow GI IXJ, UK

e-mail: monica.oliveira@strath.ac.uk

V. Faustino - D. Pinho - R. Lima

CEFT, Faculdade de Engenharia da Universidade do Porto (FEUP),

R. Dr. Roberto Frias 4200-465 Porto, Portugal

R. C. Calhelha - I. C. F. R. Ferreira

CIMO-ESA, Polytechnic Institute of Bragança, C. Sta. Apolónia 5301-857,

Bragança, Portugal

e-mail: calhelha@ipb.pt

I. C. F. R. Ferreira

e-mail: iferreira@ipb.pt

R. Lima et al. (eds.), Visualization and Simulation of Complex Flows 
degree of the deformation index (DI) of the flowing RBCs throughout the microchannel at its centerline. A suitable image analysis technique is used for semi-automatic measurements of average DIs, velocity and strain rate of the RBCs travelling in the regions of interest. The results reveal a strong deformation of RBCs under both extensional and shear stress dominated flow conditions.

\section{Introduction}

It is known that red blood cells (RBCs) at rest exhibit a biconcave disk shape $8 \mu \mathrm{m}$ in diameter and $2 \mu \mathrm{m}$ thick and comprise $40-50 \%$ of whole blood volume (Caro et al. 1979; Lima et al. 2012). An RCB at rest has a shape close to a circle but when subjected to certain flow conditions it has also the ability to deform and consequently evolve to other shapes (Dobbe et al. 2002). For example, the RBCs change to an ellipsoidal shape when submitted to shear stress and elongate significantly to pass through the smallest capillaries of the microcirculation $(3-7 \mu \mathrm{m})$ (Hardeman and Ince 1999, Dobbe et al. 2002). The RBC deformability plays a major role for oxygen delivery in the microcirculation and reduced RBC deformability is found to be linked to certain diseases such as diabetes and sickle cell anemia (Mokken et al. 1992; Hardeman and Ince 1999; Shin et al. 2007).

The clinical relevance of $\mathrm{RBC}$ deformability has been prompting the development of methods for measuring this phenomenon. Some examples are: the RBC filtration - a simple method that measures the ability of RBC to pass a filter by calculating the transit time required for the passage of a certain RBC (Gueguen et al. 1984); laser diffraction ellipsometry (ektacytometry)-based on laser diffraction analysis of RBCs under different stress levels (Mokken et al. 1992; Shin et al. 2004); rheoscopy-RBCs are deformed by simple shear flow and image analysis techniques allow a direct measurement of the RBC deformability (Dobbe et al. 2002); micropipette aspiration-RBCs are aspirated into glass capillaries with diameters of $1-5 \mu \mathrm{m}$ and the RBC deformability is calculated by using the measured negative pressure needed to aspirate the RBC (Mokken et al. 1992).

The recent developments in microfabrication make it possible to fabricate transparent micrometer-sized channels, and as a result several studies have proposed microfluidic devices able to measure the motion and dynamical deformation of cells flowing through the microchannels (Abkarian et al. 2006; Shevkoplyas et al. 2006; Zhao et al. 2006, Abkarian et al. 2008; Fujiwara et al. 2009, Kang et al. 2008; Lima et al. 2009, Tomaiuolo et al. 2011; Leble et al. 2011). Most of the proposed biomedical microdevices focus on the effect of shear flow alone. Shear flow in this context results from the flow induced by shear stress caused by the velocity gradient that develops adjacent to the neighbouring walls of the microdevice (Fig. 1b). Some examples of shear flow studies from the literature are the measurement of the RBC cellular trajectories and deformation under a transient high shear stress in a narrow channel (Zhao et al. 2006) and the determination of 
out the ised for e RBCs ation of

эе $8 \mu \mathrm{m}$ volume a circle rrm and e RBCs ate sig$;-7 \mu \mathrm{m})$ plays a I deforkle cell

2 develhe RBC filter by jueguen iser dif2; Shin 1 image (Dobbe pillaries sing the 992).

abricate ive prormation koplyas ng et al. it of the e. Shear 1 by the he mic$\geq$ are the ransient ation of

cell deformability through a single $\mathrm{RBC}$ flowing in a microfluidic device with a microchannel thickness comparable to $R B C$ size (Tomaiuolo et al. 2011). Besides the effect of shear flow, extensional flow [in which stretching flow is generated as a consequence of a velocity gradient in the flow direction (Fig. 1b), and the combination of both can be encountered in the human body. Relevant examples include micro-stenosis, which can be generated at a retinal arteriole by foam cell formation due to low-density lipoprotein (LDL) migration (Kang et al. 2008); in microvascular networks composed of small irregular vessel segments; in prosthetic blood contacting devices, such as blood pumps and other medical instrumentation, e.g. syringes. Despite the importance of the study of RBC deformation under extensional flow, only a few studies have been reported in the literature. Hence, it is crucial not only to understand the motion and $\mathrm{RBC}$ mechanical properties under the effect of simple shear flow but also to quantify the influence of extensional flow and/or the combination of both on the RBCs deformation and orientation.

Flows through contractions generate complex flows despite their simple geometries. The flow of single phase Newtonian and non-Newtonian fluids through contractions is well studied both experimentally and numerically, both at the macro and micro-scale. The flow involves a reduction in the cross-sectional area, which generates strongly converging flows as the fluid goes through the contraction. With complex fluids, the flow has been shown to exhibit a variety of flow structures, such as Moffatt vortices, lip vortices, and corner vortices, that depend on the rheological characteristics of the fluid and the geometric configuration of the contraction, including its shape (Fig. 1a-c) and configuration

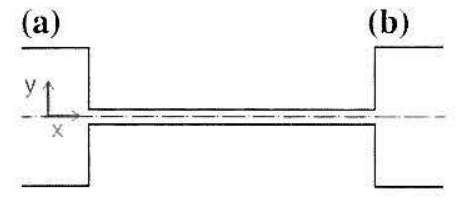

(d)

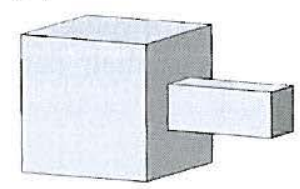

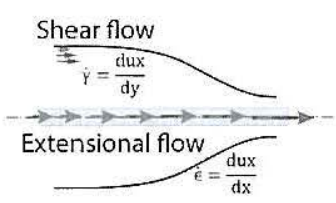

(e)

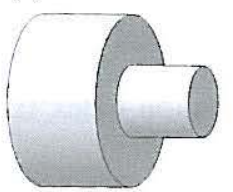

(c)

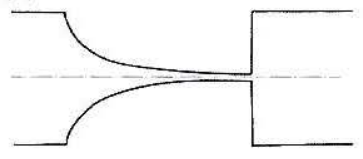

(f)

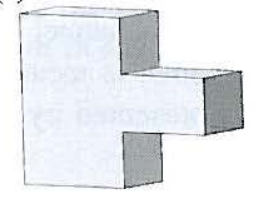

Fig. 1 Fluid flow in contractions with different shapes: a Sudden contraction b Smooth contraction c Hyperbolic contraction; and configurations: $d$ Square-square, e Axisymmetric, f Planar 2D (if AR is high), planar 3D (if AR is moderate or low). The aspect ratio (AR) is defined as $h / w$ where $h$ refers to the depth and $w$ Refers to the width of the microchannel. An illustration of the velocity gradients expected in different regions dominated by shear and extensional flow is shown in panel b) 
Fig. 2 a Illustration of the entire microchannel view. b Schematic geometry and dimensions of the hyperbolic contraction region of the microchannel (a)

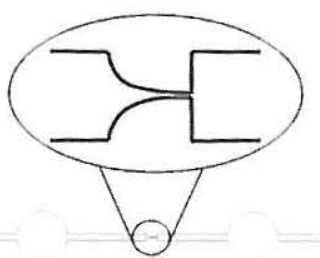

(b)

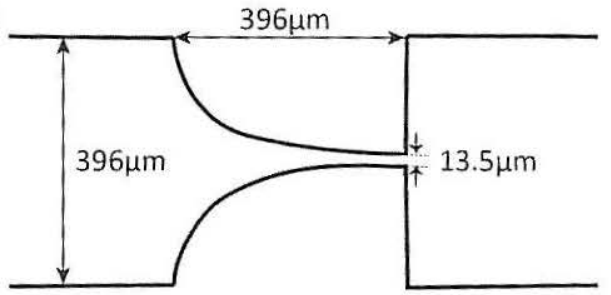

(Fig. 1d-f). The flow exhibits mixed kinematics with strong extensional flow along the centreline (stretching flow as the fluid accelerates as it is going through the contraction, Fig. 1c), and shear flow close to the walls (Fig. 1a).

Blood is an extremely complex fluid due to a suspension of different deformable cells of varying in size, such as red blood cells, white blood cells and platelets. Although the flow of single phase Newtonian and non-Newtonian fluids through contractions has been well studied, this is not the case for blood flow. Among the few are the microscopic studies performed by Zhao et al. (2006) and by Kang et al. (2008) where the authors investigated the mechanical behaviour of RBCs when they pass through sudden and smooth contraction, respectively. Others such as Lee et al. (2009), Yaginuma et al. (2011a, b, 2013) and Sousa et al. (2011) studied the effect of the extensional flow in a hyperbolic converging microchannel for animal blood, human blood and blood analogue fluids, respectively. In this study, we use a microchannel having a hyperbolic shape (cf. Fig. 2) with a very low aspect ratio $(\mathrm{AR}=0.035)$ in which the $\mathrm{RBCs}$ experiences a strong extensional flow but in addition shear flow effects are not negligible due to the low $\mathrm{AR}$ of the microchannel geometry. The RBC deformation flowing throughout this microchannel is measured along the centerline and the degree of their deformability is presented by the deformation index (DI).

\section{Materials and Methods}

\subsection{Working Fluids and Microchannel Geometry}

The working fluid used in this study was hank's balanced salt solution (HBSS) containing $2 \%$ Hct of Human RBCs. The blood was collected from a healthy adult 
Fig. 3 Experimental set-up

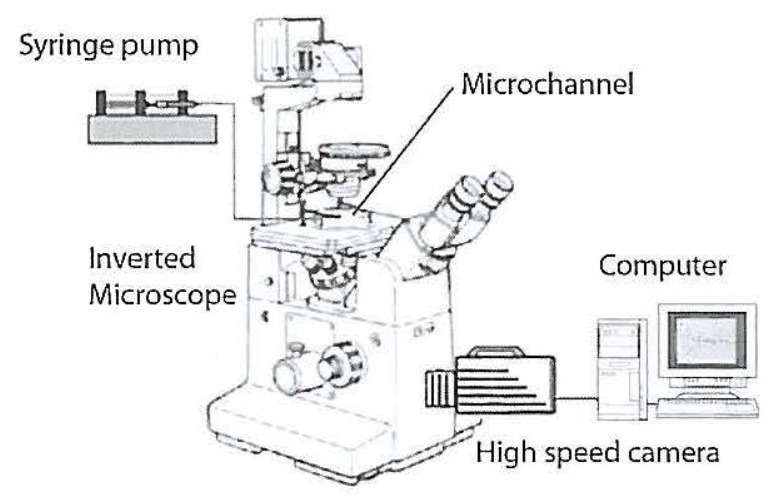

volunteer, and ethylenediaminetetraacetic acid (EDTA) was added to prevent coagulation. The RBCs were separated from the plasma and buffy coat by centrifugation $(1,000 \mathrm{rpm}$ for $10 \mathrm{~min})$. The RBCs were then washed and centrifuged with HBSS twice. Washed RBCs were diluted with HBSS to make several samples with hematocrit levels of $\sim 2 \%$ by volume. All blood samples were stored hermetically at $4{ }^{\circ} \mathrm{C}$ until the labelling.

The confocal system used in the present study consists of an inverted microscope (Diaphot 300, Nikon) combined with a high-speed camera (FASTCAM SA3, Photron). By using a soft-lithography technique we were able to manufacture a polydimethylsiloxane (PDMS) microchannel having a hyperbolic shape. As described in Fig. 2, the dimensions of the microchannel are $396 \mu \mathrm{m}$ $(w) \times 396 \mu \mathrm{m}(l) \times 14 \mu \mathrm{m}(h)$ where $w, l$ and $h$ refer to the width of the inlet microchannel, the length of the hyperbolic contraction region and the depth of the microchannel. From this, the aspect ratio $\mathrm{AR}=h / w$ can be obtained as 0.035 . The PDMS microchannel was placed on the stage of the microscope where the flow rate $Q$ of the working fluids was kept constant $(0.5 \mu 1 / \mathrm{min})$ by means of a syringe pump (PHD ULTRA) with a $1 \mathrm{~mL}$ syringe (TERUMO SYRING).

The images of the flowing RBCs were captured using the high speed camera at a frame rate of 7,500 frames/s and were then transferred to the computer to be analyzed. An illustration of the experimental set-up is shown in Fig. 3.

\subsection{Image Analysis}

High magnification was used to obtain better quality images so that the accuracy of $\mathrm{RBC}$ measurements is maximized during image analysis. As can be seen in Fig. $4 a-c$, the original data consists of three sets of video sequences focusing in different regions of the channel. These sets of images can be combined to obtain the required in information the entire hyperbolic contraction region of the microchannel as shown in Fig. 4d. 
Fig. 4 Original video images (a), (b), and (c) focusing on different regions of the channel, and a combined image (d) that presents the centreline region $(y=0)$ at the hyperbolic microchannel

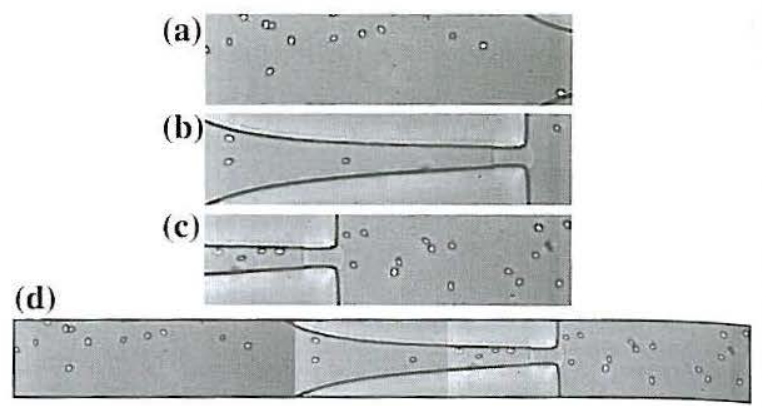

The following image handling was carried out. First, the captured video data was converted to JPEG image sequences. Each image has 1,024 $\times 256$ pixels size and in total 4,072 frames were obtained for one video with a frame interval of $133 \mu$ s. The sequences of static images (known as a stack) were processed using the image handling software ImageJ (Abramoff et al. 2004). For measuring flowing RBC particles, the following pre-processing was executed on the stack. First, an averaged image (background) was created from the original stack images by averaging each pixel over the sequence of static images, and then subtracted from the stack images. This process eliminates all the static objects, for example, the microchannel walls and other static artefacts, leaving only RBCs visible. Next, the images were filtered by replacing each pixel with the median of the neighbouring pixel values with a mask size of $3 \times 3$ pixels, in order to reduce the artefacts and enhance the image quality. Then the brightness and contrast of the resulting images were manually adjusted. Finally, the grey scale images were converted to binary images adjusting the threshold level. The well-known Otsu threshold method was used in this case (Otsu 1979). The final images contain regions of interest (RBCs) as black ellipsoidal objects against a white background. After this segmentation process, the flowing RBCs were measured frame by frame automatically by Analyze Particles function in ImageJ. This function counts and measures the objects in binary images, by detecting the edge of the objects and outlining them. Various parameters, such as area and circularity of the cells, were appropriately pre-set so that the values outside the range specified were ignored. For instance, we set the range of area as $17-150 \mu \mathrm{m}^{2}$ such that the objects smaller than $17 \mu^{2}$ and larger than $150 \mu \mathrm{m}^{2}$ were eliminated from the results table. Likewise, the circularity range was set to be $0.5-1.0$. Here the circularity is defined as $4 \pi \times($ area $) /(\text { perimeter })^{2}$. This way, most of the apparent deviant objects (e.g. out-of-focus cells, aggregated cells, etc.) were ignored in the measurements. The most important steps of image handling described above are illustrated in Fig. 5.

The major output results of this measurement are the major (primary) and minor (secondary) axis lengths of the ellipse that can be best fitted to the RBCs and the $x-y$ coordinates of their centroid. Using this set of data, the deformation index (DI) of all the measured cells was calculated using Matlab and stored together with the cell positions (centroid) given by their $x-y$ coordinates. In this 
Fig. 5 Steps of image analysis. a Original image, b Binary image, c A view of cells automatically measured by Image J function Analyze Particles (a)

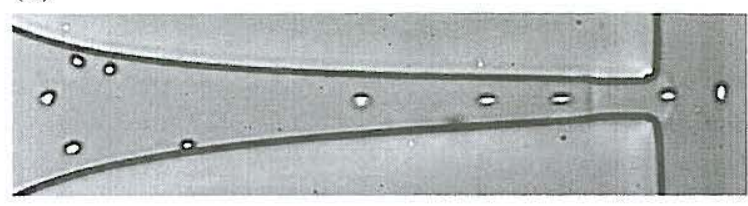

(b)

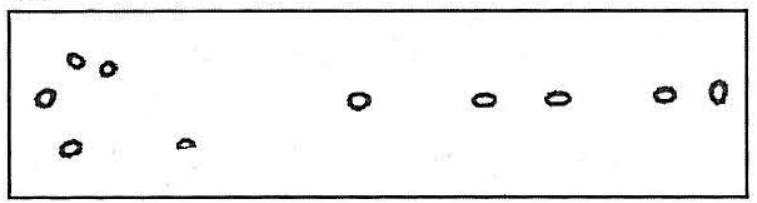

(c)

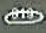

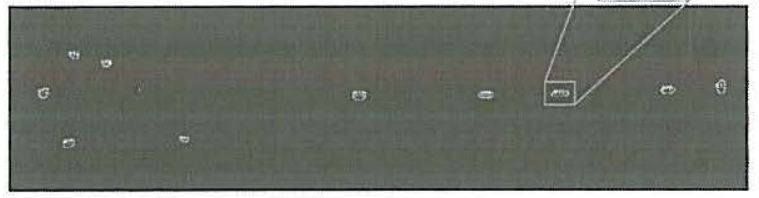

study, DI is defined by the formula shown in Fig. 6, where $\mathrm{X}$ and $\mathrm{Y}$ refer to the major and minor axis lengths of the cell, respectively. RBC deformability is the ability of the erythrocytes to deform when submitted to certain flow conditions in both in vivo and in vitro environments, and the deformation index can display the degree of their deformability with values between 0 and 1 , where 0 indicates a perfect circle and higher values represent more elongated cells.

In addition to the DI measurement, the trajectories of several flowing RBCs at a flow rate of $0.5 \mu \mathrm{l} / \mathrm{min}$ were measured manually using MTrackJ plug-in installed in ImageJ (Meijering et al. 2012). MTrackJ provides useful functions for tracking objects in motion. By placing the target $\mathrm{RBC}$ within the snap window, it computes automatically the centroid of the RBC based on Otsu threshold method. We manually follow the identified $\mathrm{RBC}$ frame by frame recording the centroid trajectory, and finally get the velocity of the RBC. By using real velocity of the RBCs the strain rate was also calculated.

\section{Results and Discussion}

\subsection{Deformation Index (DI) of RBCs in Extensional Flow}

In this work, the deformation of the RBCs travelling through the microchannel centerline $(y=0)$ region was observed. As stated in the previous section, the original data consists of three video sequences. Therefore, the DI values are the 


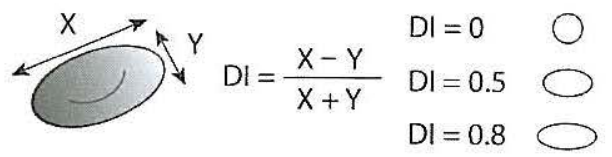

Fig. 6 Definition of deformation index. $X$ and $Y$ refer to the major and minor axis lengths of the cell. Note that the length of major axis $(X)$ oriented to the flow direction considers the cell's travel length caused by the camera's exposure time

average of pre-defined regions of interest along the centreline at each image. Each region of interest is defined as $36 \mu \mathrm{m}$ long and $20 \mu \mathrm{m}$ high and located consecutively from far upstream to far downstream of the contraction part of the microchannel (see Fig. 7a). All the cells captured in each region were measured and the averaged DI was calculated. Fig. $7 \mathrm{~b}$ presents average DI through the microchannel for an inlet flow rate of $0.5 \mu \mathrm{l} / \mathrm{min}$. The horizontal axis corresponds to the microchannel $x$ axis where $x=0$ is set to be at the beginning of the hyperbolic curve and $x=1$ is set to be at the end of the contraction region. It is possible to observe that the DI values at the centreline far upstream of the contraction are around 0.13 and fairly constant. After entering the contraction region, RBCs start elongating and their DI values start increasing. At the region right before the exit of contraction part $(x=1)$, the DI reaches the maximum value of 0.33 (see Figs. $7 \mathrm{~b}, 8$ ). Just downstream of the expansion plane DI drops dramatically and the

Fig. 7 Evaluation of the deformation of the flowing $\mathrm{RBCs}$ around the centerline of the hyperbolic microchannel. a Illustration of the regions of interest to analyze the RBCs deformation index (DI). Note that each region is $36 \mu \mathrm{m}$ long and $20 \mu \mathrm{m}$ high. b Average DI in each region throughout the microchannel at a flow rate of $0.5 \mu \mathrm{l} / \mathrm{min}$ (a)
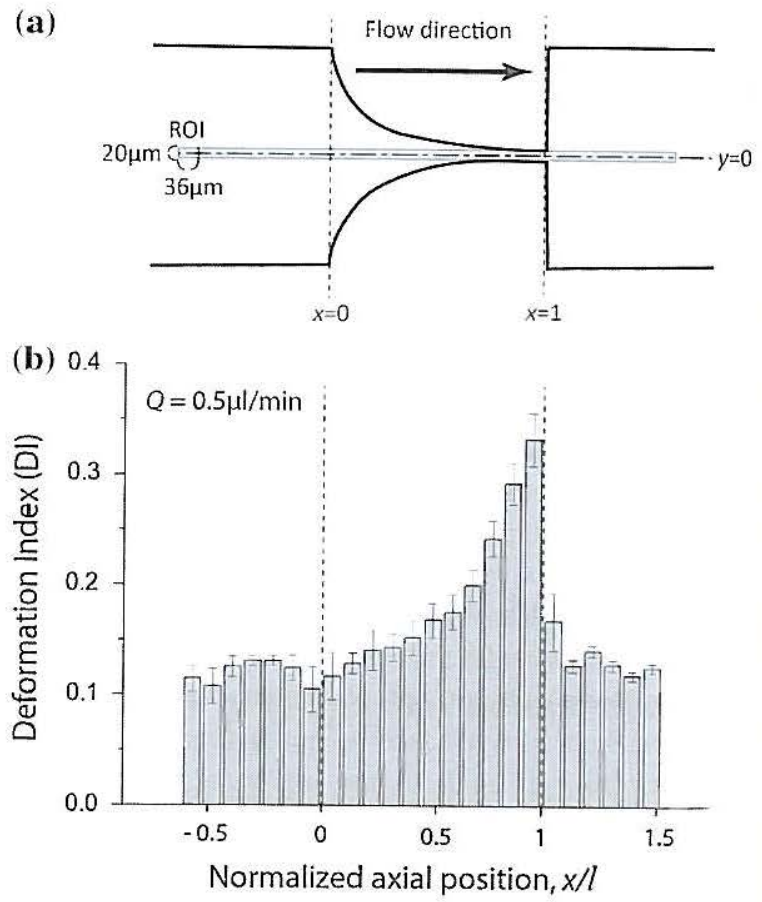
Fig. 8 Evolution of $\mathrm{RBC}$ deformation throughout the hyperbolic microchannel

ze. Each consecthe mired and $\because$ microIs to the perbolic ssible to tion are iCs start the exit .33 (see ' and the

hyperbolic microchannel channel.

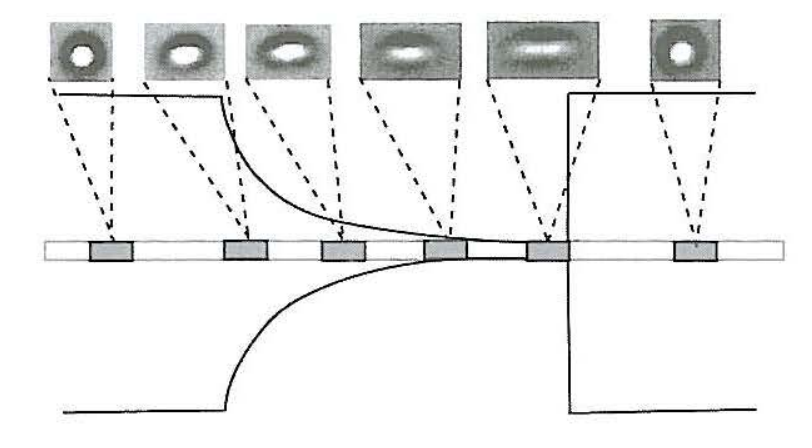

RBCs start returning to their original shape. It can be seen that the DI value far downstream in this plane is almost the same as the one at the upstream of the

\subsection{Flow Rate Effect on RBCs Deformation Index (DI)}

Figure 9 shows the RBCs DI in the expansion region for three different flow rates, i. e., $0.010,0.5$ and $1.0 \mu 1 / \mathrm{min}$ in the same microchannel. Following the same measurement procedures used before, these results (see Fig. 9) shows that for all the flow rates studied the DI has a maximum value at the region right before the exit of the contraction. After that, the RBCs tend to recover their initial shape which corresponds to a minimal value of the DI attained when they are no longer exposed to extensional flow. Another additional result is that the RBCs have a tendency to increase the DI as the flow rate increases (Yaginuma et al. 2013). In contrast, in the work performed by Zhao et al. (2006), where shear flow dominates (see Fig. 1), the DI reaches a maximum value at a shear rate of $1,760 \mathrm{~s}^{-1}$ and the cells could not deform any further, regardless the increase of the flow rate. These qualitative comparisons suggest that the flow rate strongly affects the response of RBCs when exposed to an extensional flow field. Nevertheless, to confirm it, further careful studies should be necessarily employed with higher flow rates and with different dimensions of the microchannels.

\subsection{Axial Velocity and Strain Rate}

To measure the actual RBC velocity, the cells traveling along the centerline of the microchannel were manually tracked using ImageJ plug-in, MTrackJ. Along with the velocity for each tracking point, the strain rate was also calculated from $\mathrm{d} v / \mathrm{d} x$, where $\mathrm{d} v$ refers to differential cell velocity and $d x$ refers to the distance between two tracking points. 
Fig. 9 DI recovery in the expansion region for different flow rates: $\mathbf{a} \mathrm{Q}=0.010 \mu \mathrm{l} /$ $\min , \mathrm{b} Q=0.5 \mu \mathrm{l} / \mathrm{min}$, c $\mathrm{Q}=1.0 \mu \mathrm{l} / \mathrm{min}$

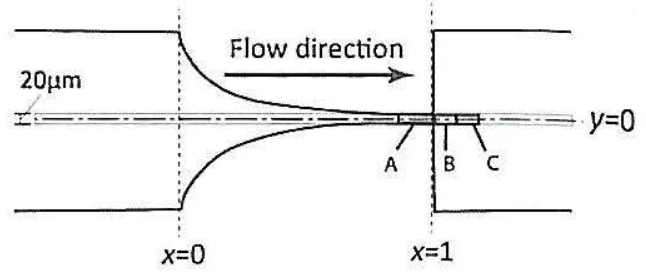

(a)

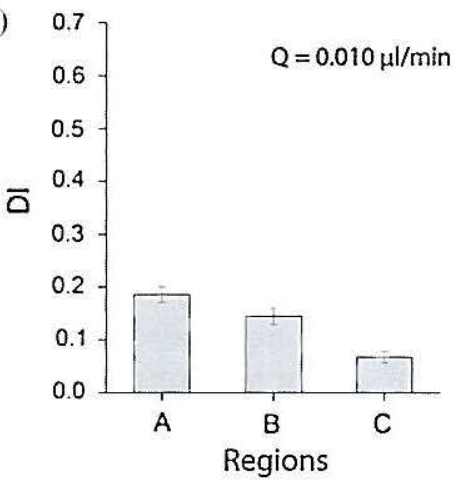

(b)
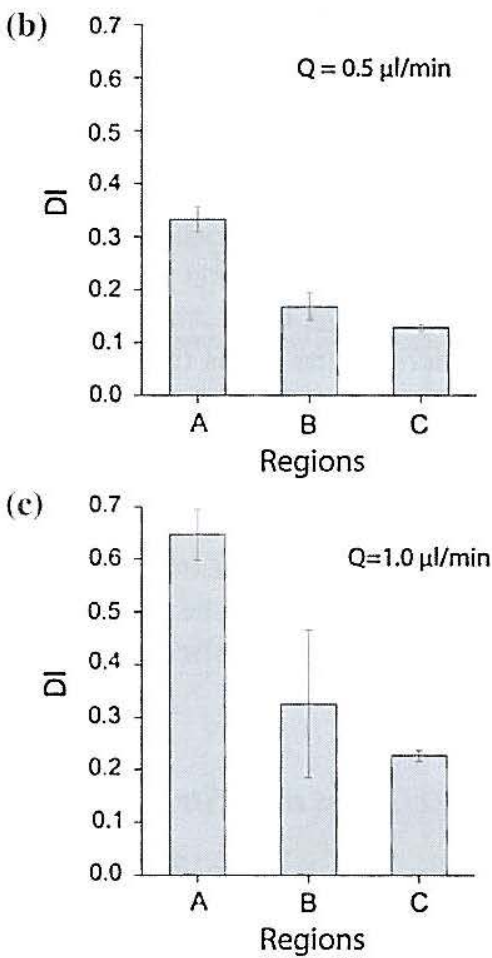


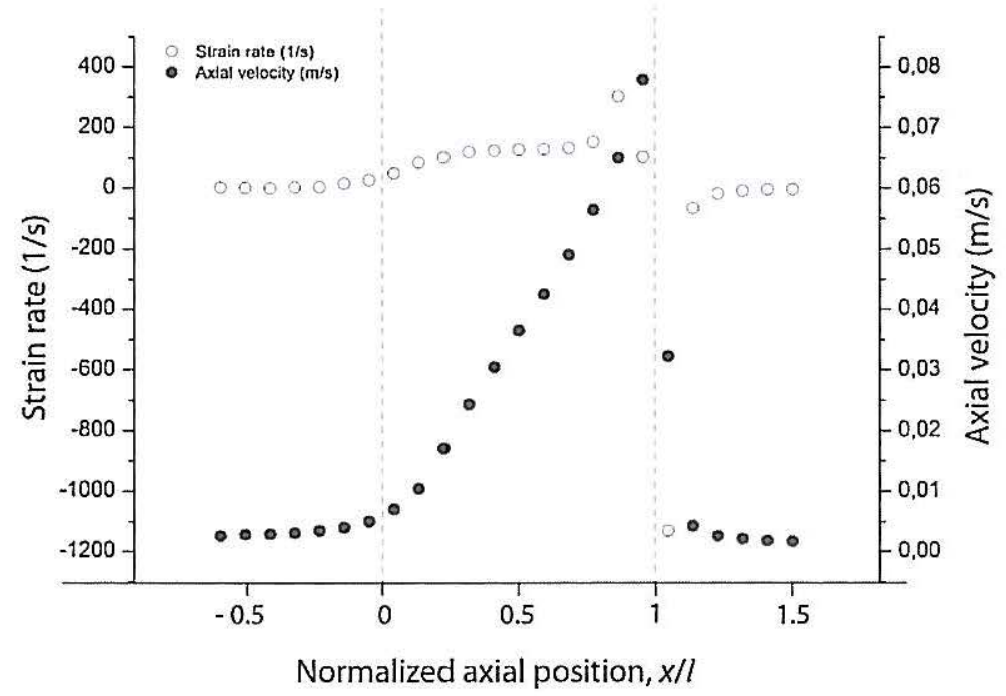

Fig. 10 Average axial velocity at the centreline and corresponding strain rate for RBCs flowing at the flow rate of $0.5 \mu \mathrm{l} / \mathrm{min}$

Figure 10 shows the average cell velocity and average strain rate for every $36 \mu \mathrm{m}$ axial region throughout the central region of the microchannel at a flow rate of $0.5 \mu \mathrm{l} / \mathrm{min}$. The velocity of the cells far upstream is initially constant and then starts slightly increasing when the cells approach the entrance of the hyperbolic region. After the entrance $(x=0)$, the velocity increases almost linearly, corresponding to a constant strain-rate, and reaches the maximum value at the end part of the contraction region. After the cells are released from the contraction region, their velocity decreases and eventually recovers the upstream velocity value. The fact that we are able to obtain a wide region of constant strain-rate confirms the potential of hyperbolic channels for generating a controlled and strong homogeneous extensional flow field.

\section{Conclusions and Future Directions}

The current study evaluated the RBC deformability in the relatively low aspect ratio hyperbolic contraction microchannel, where the RBCs experience a strong extensional and shear flow. The results show that when the RBCs enter the hyperbolic contraction region they tend to elongate up to a maximum value indicating their high deformability in a strong extensional flow. For a flow rate of $0.5 \mu \mathrm{l} / \mathrm{min} \mathrm{RBC}$ deformation index increases from about 0.12 to 0.33 with a maximum strain rate up to $320 \mathrm{~s}^{-1}$. Qualitative comparison between DIs of sudden 
contraction and hyperbolic contraction microchannels indicated that for latter case the flow rate strongly affects the response of RBCs under extensional flow induced deformation. These results support the use of hyperbolic converging microchannels as a new promising approach for RBC deformability assessment for clinical applications. We recognize that the working fluid used in the current study (HBSS with $2 \% \mathrm{Hct}$ of human blood) is not close to in vivo environment. However, our purpose is to develop an alternative diagnostic microfluidic system. Therefore, it is important to notice these differences might affect the RBC deformability but the efficiency of deformability measurements have to be considered. For example, low Hct is necessary for better image analysis results. HBSS (physiological saline) is used as it is closer to plasma than the other possible solutions such as Dextran 40.

Acknowledgments The authors acknowledge the financial support provided by: International Doctoral Program in Engineering, from the Ministry of Education, Culture, Sports, Science and Technology of Japan (MEXT), 2007 Global COE Program "Global Nano-Biomedical Engineering Education and Research Network", Japan. Grant-in-Aid for Science and Technology (PTDC/SAU-BEB/108728/2008, PTDC/SAU-BEB/105650/2008 and PTDC/EME-MFE/099109/ 2008) from the Science and Technology Foundation (FCT), FEDER and COMPETE, Portugal.

\section{References}

Abkarian M, Faivre M, Stone H (2006) High-speed microfluidic differential manometer for cellular-scale hydrodynamics. PNAS 103(3):538-542

Abkarian M, Faivre M, Horton R, Smistrup K, Best-Popescu CA, Stone HA (2008) Cellular-scale hydrodynamics. Biomed Mater 3:034011

Abramoff MD, Magalhães PJ, Ram SJ (2004) Image processing with ImageJ. Biophotonics Int $11: 36-42$

Caro C, Pedley T, Schroter R, Seed W (1979) The mechanics of the circulation. Oxford University Press, Oxford

Dobbe JGG, Hardeman MR, Streekstra GJ, Strackee J, Ince C, Grimbergen CA (2002) Analyzing red blood cell-deformability distributions. Blood Cells Mol Dis 28:373-384

Fujiwara H, Ishikawa T, Lima R, Matsuki N, Imai Y, Kaji H. Nishizawa M. Yamaguchi T (2009) Red blood cell motions in a high hematocrit blood flowing through a stenosed micro-channel. J Biomech 42:838-843

Gueguen M, Bidet JM, Durand F. Driss F, Joffre A, Genetet B (1984) Filtration pressure and red blood cell de-formability: evaluation of a new device: erythrometre. Bio-rheol Suppl $1: 261-265$

Hardeman MR, Ince C (1999) Clinical potential of in vitro measured red cell deformability, a myth? Clin Hemorheol Microcirc 21:277-284

Kang M, Ji HS, Kim KC (2008) An in-vitro investigation of RBCs flow characteristics and hemodynamic feature through a microchanel with a micro-stenosis. Int J Biol Biomed Eng 1(2): $1-8$

Leble V, Lima R, Dias R, Fernandes C, Ishikawa T, Imai Y. Yamaguchi T (2011) Asymmetry of red blood cell motions in a microchannel with a diverging and converging bifurcation. Biomicrofluidics 5:044120

Lee SS, Yim Y, Ahn KH, Lee SJ (2009) Extensional flow-based assessment of red blood cell deformability using hyperbolic converging microchannel. Biomed Microdevices $11: 1021-1027$ 
atter case $\rightarrow$ induced icrochanr clinical y (HBSS ever, our fore, it is $y$ but the nple, low saline) is xtran 40.

ternational cience and 3iomedical echnology EE/099109/ Portugal.

ometer for Ilular-scale เotonics Int m. Oxford Analyzing hi T (2009) ro-channel. ure and red reol Suppl mability, a sristics and iomed Eng ymmetry of bifurcation. blood cell icrodevices
Lima R, Oliveira MSN, Ishikawa T, Kaji H. Tanaka S, Nishizawa M, Yamaguchi T (2009) Axisymmetric PDMS microchannels for in vitro haemodynamics studies. Biofabrication 1(3):035005

Lima R, Ishikawa T, Imai Y, Yamaguchi T (2012) Blood flow behavior in microchannels: past, current and future trends. In: Dias R, Martins AA, Lima R et al (eds) Single and two-phase flows on chemical and biomedical engineering. Bentham, Oak Park, pp 513-554

Meijering E, Dzyubachyk O, Smal I (2012) Methods for Cell and Particle Tracking. Methods Enzymol 504:183-200

Mokken FC, Kedaria M, Henny CP, Hardeman MR, Gelb AW (1992) The clinical importance of erythrocyte deformability, a hemorheological parameter. Ann Hematol 64:113-122

Otsu N (1979) A threshold selection method from gray-level histograms. IEEE Trans Sys Man Cyber 9(1):62-66

Shevkoplyas SS, Yoshida T, Gifford SC, Bitensky MW (2006) Direct measurement of the impact of impaired erythrocyte deformability on microvascular network perfusion in a microfluidic device. Lab Chip 6(7):914-920

Shin S, Ku Y, Park MS, Suh JS (2004) Measurement of red cell deformability and whole blood viscosity using laser-diffraction slit rheometer. Korea-Australia Rheol J 16:85-90

Shin S, Ku Y, Ho J, Kim Y, Suh J, Singh M (2007) Progressive impairment of erythrocyte deformability as indicator of microangiopathy in type 2 diabetes mellitus. Clin Hemorheol Micro 36:253-261

Sousa PC, Pinho FT, Oliveira MSN, Alves M (2011) Extensional flow of blood analog solutions in microfluidic devices. Biomicrofluidics 5:014108

Tomaiuolo G, Barra M, Preziosi V, Cassinese A, Rotoli B, Guido S (2011) Microfluidics analysis of red blood cell membrane viscoelasticity. Lab Chip 11:449-454

Yaginuma T, Lima R, Oliveira MSN, Ishikawa T, Yamaguchi T (2011a) Flow of red blood cells through a microfluidic extensional device: an image analysis assessment. In: Proceedings of the III ECCOMAS thematic conference on computational vision and medical image processing: VipIMAGE, pp 217-220

Yaginuma T, Oliveira MSN, Lima R, Ishikawa T, Yamaguchi T (201/b) Red blood cell deformation in flows through a PDMS hyperbolic microchannel. Proc NSTI-Nanotech 2:505-507

Yaginuma T, Oliveira MSN, Lima R, Ishikawa T, Yamaguchi T (2013). Human red blood cell behavior under homogeneous extensional flow in a hyperbolic-shaped microchannel. Biomicrofluidics 7:054110

Zhao R, Antaki JF, Naik T, Bachman TN, Kameneva MV, Wu ZJ (2006) Microscopic investigation of erythrocyte deformation dynamics. Biorheology 43(6):747-765 
Lecture Notes in Computational Vision and Biomechanics 12

Rui Lima · Yohsuke Imai - Takuji Ishikawa - Mónica S. N. Oliveira Editors

\section{Visualization and Simulation of Complex Flows in Biomedical}

\section{Engineering}

This book focuses on the most recent advances in the application of visualization and simulation methods to understand the flow behavior of complex fluids used in biomedical engineering and other related fields. It shows the physiological flow behavior in large arteries, microcirculation, respiratory systems and in biomedical microdevices.

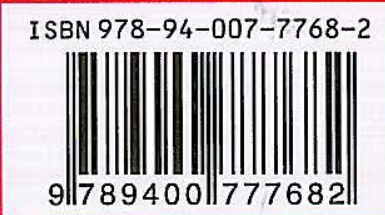

$>$ springer.com 